\title{
Adaptive and Robust Control Method with Estimation of Rolling Characteristics for Looper Angle Control at Hot Strip Mill
}

\author{
Hideki ASADA, Akira KITAMURA, ${ }^{1)}$ Satoshi NISHINO ${ }^{1)}$ and Masami KONISHI ${ }^{2)}$ \\ Kobe University, Rokkoudaicho, Nada-ku, Kobe, Hyogo Japan. \\ 1) Electronics Research Laboratory, Kobe Steel Ltd., \\ Takatsukadai, Nishi-ku, Kobe, Hyogo 651-2271 Japan. \\ 2) Faculty of Engineering, Okayama University, Tsushima-naka,
} Okayama 700-8530 Japan.

(Received on July 1, 2002; accepted in final form on November 1, 2002)

\begin{abstract}
In this paper, an adaptive robust control method for looper height control of hot strip finishing mill is proposed. The change in strip tension, which exerts an influence on the width and thickness of the products, is induced by the looper height variation. Accordingly, it is important to control the looper height precisely. The looper control system may suddenly become unstable due to a change in the characteristics of rolled material and an occurrence of the slip between roll and strip while rolling. In order to maintain the control system stable even under these changes, the robust control system has been developed. However, there remained problems of slow response of the robust controller. The reason is that the control gain is set at a low value to assure the stability for the various kinds of disturbances. To solve the problem, it is necessary to set at the appropriate control gain. In this paper, the estimation of a damping coefficient of the looper height control system is carried out to adapt the control gain according to the damping coefficient. The proposed adaptive controller assures both the robustness and a high control performance regardless of the change in rolling conditions and disturbances. Using this method, the looper height control can guarantee both the stability of the looper height control and the high degree of accuracy in control.
\end{abstract}

KEY WORDS: adaptive control; parameter estimation; robust control; internal model control; looper control system; hot strip mill.

\section{Introduction}

In hot strip mill, the variations of strip tension between rolling stands exert an influence on the width and thickness of the product. The strip tension changes in response to the looper height. When the tension momentarily changes, the looper moves and compensates for the change in the tension. So, and it is important to control the looper height precisely. As for the looper height control method, PI control methods and multivariable control methods have been studied. ${ }^{1-4)}$ However, these conventional looper control systems may suddenly become unstable due to the change in the characteristics of rolled material and the slip between roll and strip while rolling. To overcome the problem, the robust control method has been proposed.,6) The conventional robust control system is designed so as to maintain the stability even under the wide change in the characteristics of rolled material and the rolling conditions. Because the control gain of the conventional robust controller is determined by considering the worst disturbance, there exists a problem of slow response of the robust controller. The conventional adaptive control system ${ }^{7)}$ is designed on the assumption that the parameter can be estimated accurately. So it cannot guarantee the system stability if the estimated error of the parameter exists. To solve these problems, an adaptive robust control method accompanying the estimation of the looper parameters has been proposed. The con- trol gain of the proposed method can be changed adaptively by the estimation of the looper parameter. And the robust stability is theoretically studied by the sensitivity and the complementary sensitivity in order to guarantee the system stability in spite of existing the estimated error of the parameter. Using this method, the looper height control can guarantee both the stability of looper height dynamics and the high degree of accuracy in control simultaneously.

In this paper, the looper height model is explained first. Second, the estimation of looper parameters related to looper height dynamics is explained. Then the construction of the adaptive robust controller is explained. Finally, the effect of the proposed method is checked by numerical simulations.

\section{The Looper Height Model}

The looper height model of hot strip mill consists of factors that are changed by the slip between roll and strip, characteristics of the rolled material and so on. However, it is difficult to measure exactly the Young ratio of material and the friction between the roll and the strip. Accordingly, the mathematical model for the looper height is constructed based on the input/output responses. The control input of the looper height system is the reference value of the velocity to the mill motor and the controlled variable is the looper angle (Fig. 1). 


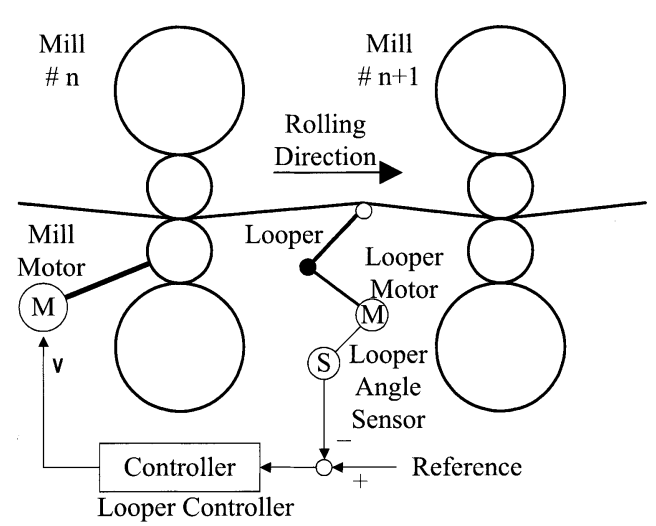

Fig. 1. Looper control system of hot strip mill.

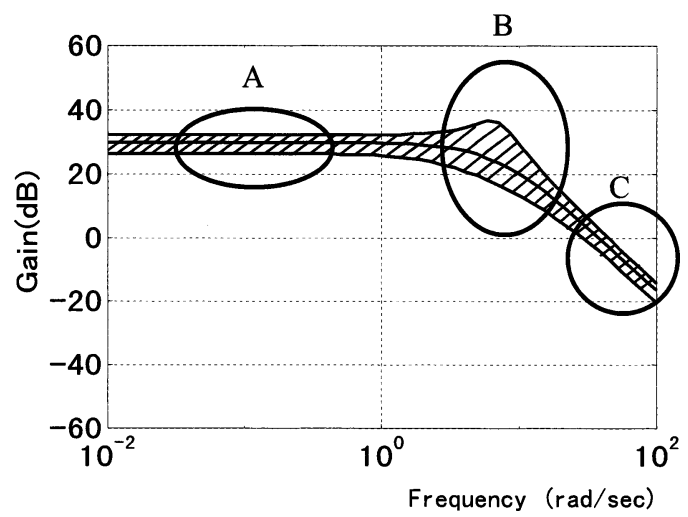

Fig. 2. Measured gain diagrams of looper control system.

Fujisaki et al. proposed an experimental looper height model which approximates loop dynamics by using second order lag element with dead time using data of input and output signals. ${ }^{5,6)}$ Here, the same approach is made to describe the looper model is written as follows:

$$
\frac{Y(s)}{U(s)}=\frac{G \omega_{n}^{2}}{s^{2}+2 \zeta \omega_{n} s+\omega_{n}^{2}} e^{-L s}
$$

Where, $U$ is control input that is the reference value of the velocity, $Y$ is controlled variable and it's the looper angle, $s$ is the Laplace transform parameter, $G$ is the stationary gain, $\omega_{n}$ is the resonant frequency, $\zeta$ is the damping coefficient, and $L$ is the dead time.

The results of the analysis using large amounts of data, which were measured under various rolling conditions, are shown in Fig. 2. As for the data, it is confirmed that the damping coefficient was changed largely (see zone B in Fig. 2) and contrary to this, both the stationary gain and the resonant frequency were remained almost constant (see zone $\mathrm{A}$ and $\mathrm{C}$ in Fig. 2).

\section{Estimation of the Damping Coefficient}

As shown in Fig. 2, the damping coefficient that influenced the stability of the looper height control system greatly was changed. Therefore, the estimation of the damping coefficient is made to adapt the robust control gain for the compensation of the change in the looper characteristics.

The damping coefficient is estimated by the recursive

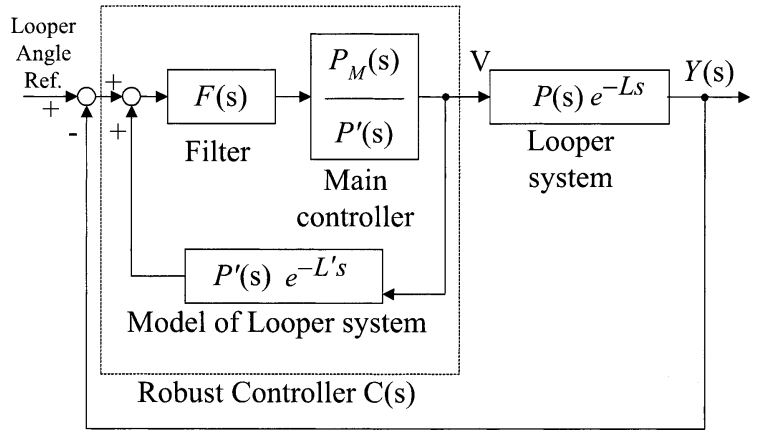

Fig. 3. Structure of internal model control.

least squares method. ${ }^{7,8)}$

When input/output signals are stationary and the amplitudes of these signals are sufficiently small, there may occur a problem that the estimation error in the damping coefficient increases. To overcome this problem, $J$ indicating the reliability of the estimation is calculated by the Eq. (2). The evaluation value $J$ is the sum of the differences in the looper angle changes calculated in every sampling time. The value of $J$ increases when the damping coefficient change to small and the looper angle becomes fluctuated. When the evaluation value $J$ becomes larger than the threshold value, determined beforehand considering the past measured data, the estimated result is judged to be trustworthy. ${ }^{7)}$

$$
J[i]=\lambda \cdot J[i-1]+|(Y[i]-Y[i-1])-(Y[i-1]-Y[i-2])|
$$

Where, $\lambda$ is a forgetting coefficient and $Y[i]$ is the looper angle at the sampling time $i$.

\section{An Adaptive Robust Controller}

The Internal Model Control (IMC) is one of the predictive control method and general for process control. The IMC can be designed based on the desirable relation between input and output even if the model error exists. ${ }^{9)}$ So we design the structure of our proposed controller based on the IMC. The block diagram of the IMC is shown in Fig. 3. ${ }^{6)}$

Where $C(s), F(s)$ and $P_{M}(s)$ are the transfer functions of the controller, the filter model and the reference model respectively. And $P^{\prime}(s) e^{-L^{\prime} s}$ and $P(s) e^{-L s}$ are the model and transfer function of the dynamics of the looper system respectively.

The transfer function $C(s)$ can be expressed as follows ${ }^{6}$ :

$$
C(s)=\frac{F(s) P_{M}(s)}{P^{\prime}(s)\left\{1-F(s) P_{M}(s) e^{-L^{\prime} s}\right\}}
$$

The design items for the system in Fig. 3 are $F(s)$ and $P_{M}(s)$. In order to maintain the system stability even under changing the characteristics of rolled material and that of the rolling condition, $F(s)$ is designed so that the looper height control system can guarantee the robust stability by using the estimation of the damping coefficient of looper height control system. 


\subsection{Robust Stability Problem}

The complementary sensitivity function $T(s)$, which is an index of stability margin, can be expressed as follows ${ }^{10,11)}$. (see Appendix A)

$$
T(s)=\frac{F(s) P_{M}(s) P(s) e^{-L s}}{P^{\prime}(s)\left\{1-F(s) P_{M}(s) e^{-L^{\prime} s}\right\}+F(s) P_{M}(s) P(s) e^{-L s}}
$$

To maintain its robust stability, $T(s)$ is needed to be sufficiently small at all frequency when the looper height characteristics $P(s) e^{-L s}$ is changed. That is to say, $T(s)$ is designed to be sufficiently small at the frequency of the worst disturbance. In order to satisfy this condition, the characteristics $F(s)$ is to be designed sufficiently small for the worst disturbance. That is, this design is not necessary for all frequency, and it is necessary only for the frequency of low damping coefficient. However, $F(s)$ is usually designed to be small for all frequency by the conventional robust control. ${ }^{5,6)}$ For these reasons, the conventional robust control method guarantees the stability of the system, but it weakens the control performance.

From this background, an adaptive looper height robust control accompanying estimation of the damping coefficient is proposed. The adaptive control system were expected to guarantee the stability of the system and a high degree of accuracy even if the looper characteristics $P(s) e^{-L s}$ is changed. For this purpose, in the adaptive robust control system, the estimation of damping coefficient is employed. Further, the adaptive robust control system involves the reference model to satisfy the request for the response of looper angle.

The reference model $P_{M}(s)$ is designed to have the second order lag element, and the filter $F(s)$ is designed to have the first order lag element as follows:

$$
\begin{array}{r}
P_{M}(s)=\frac{\omega_{M}^{2}}{s^{2}+2 \zeta_{M} \omega_{M} s+\omega_{M}^{2}} \\
F(s)=\frac{1}{1+T_{f} s} \ldots \ldots \ldots \ldots
\end{array}
$$

where $\omega_{M}$ and $\zeta_{M}$ are designed to satisfy the request for the response of looper angle. The problem at this stage is the design of the time constant $T_{f}$ to guarantee the system stability.

The complementary sensitivity function value $|T(s)|$ can be derived out as follows:

$$
\begin{aligned}
& |T(s)|=\frac{\omega_{M}^{2} \omega_{n}^{2} \sqrt{\left(\omega_{n}^{\prime 2}-\omega^{2}\right)^{2}+\left(2 \zeta^{\prime} \omega_{n}^{\prime} \omega\right)^{2}}}{\sqrt{a^{2}+b^{2}}} \ldots \ldots . . \\
a= & \omega_{n}^{\prime 2}\left\{\left(\omega_{M}^{2}-\omega^{2}\right)\left(\omega_{n}^{2}-\omega^{2}\right)-4 \zeta_{M} \omega_{M} \zeta \omega_{n} \omega^{2}\right. \\
& \left.-T_{f} \omega\left[2 \zeta_{M} \omega_{M} \omega\left(\omega_{n}^{2}-\omega^{2}\right)+2 \zeta \omega_{n} \omega\left(\omega_{M}^{2}-\omega^{2}\right)\right]\right\} \\
& -\omega_{n}^{\prime 2} \omega_{M}^{2}\left[\left(\omega_{n}^{2}-\omega^{2}\right) \cos \left(L^{\prime} \omega\right)+2 \zeta \omega_{n} \omega \sin \left(L^{\prime} \omega\right)\right] \\
& +\omega_{n}^{2} \omega_{M}^{2}\left[\left(\omega_{n}^{\prime 2}-\omega^{2}\right) \cos (L \omega)+2 \zeta^{\prime} \omega_{n}^{\prime} \omega \sin (L \omega)\right] \\
b= & \omega_{n}^{\prime 2}\left\{T_{f} \omega\left[\left(\omega_{M}^{2}-\omega^{2}\right)\left(\omega_{n}^{2}-\omega^{2}\right)-4 \zeta_{M} \omega_{M} \zeta \omega_{n} \omega^{2}\right]\right.
\end{aligned}
$$

$$
\begin{aligned}
& \left.+2 \zeta_{M} \omega_{M} \omega\left(\omega_{n}^{2}-\omega^{2}\right)+2 \zeta \omega_{n} \omega\left(\omega_{M}^{2}-\omega^{2}\right)\right\} \\
& -\omega_{n}^{\prime 2} \omega_{M}^{2}\left[-\left(\omega_{n}^{2}-\omega^{2}\right) \sin \left(L^{\prime} \omega\right)+2 \zeta \omega_{n} \omega \cos \left(L^{\prime} \omega\right)\right] \\
& +\omega_{n}^{2} \omega_{M}^{2}\left[-\left(\omega_{n}^{\prime 2}-\omega^{2}\right) \sin (L \omega)+2 \zeta^{\prime} \omega_{n}^{\prime} \omega \cos (L \omega)\right]
\end{aligned}
$$

where $\omega_{n}^{\prime}$ and $\zeta^{\prime}$ are resonant frequency and damping coefficient of the looper height model.

The time constant $T_{f}$ of the filter $F(s)$ is designed to satisfy Eqs. (8) and (9).

$$
\begin{gathered}
|T(s)|<\beta(\omega) \\
\beta(\omega)<\frac{1}{|\Delta(\omega)|} .
\end{gathered}
$$

where $\Delta(\omega)$ is the additive uncertainty of the looper height model and can be expressed as follows:

$$
P(s) e^{-L s}=P^{\prime}(s) e^{-L^{\prime} s}+\Delta(\omega)
$$

In Eq. (10), $P(s) e^{-L s}$ shows the dynamics of the exact plant, which may contain changes of parameters.

Referring to Eq. (7), the Eq. (8) can be expressed as the second order inequality in terms of $T_{f}$ and written as follows: (see Appendix B)

$$
\begin{array}{r}
\beta^{2}\left(p_{1}^{2}+r_{1}^{2}\right) T_{f}^{2}+2 \beta^{2}\left(p_{1} q_{1}+r_{1} s_{1}\right) T_{f}+\beta^{2}\left(q_{1}^{2}+s_{1}^{2}\right) \\
-\left(\omega_{M}^{2} \omega_{n}^{2}\right)^{2}\left\{\left(\omega_{n}^{\prime 2}-\omega^{2}\right)^{2}+\left(2 \zeta^{\prime} \omega_{n}^{\prime 2} \omega\right)^{2}\right\}>0
\end{array}
$$

The second order coefficient in Eq. (11), $\beta^{2}\left(p_{1}^{2}+r_{1}^{2}\right)$, is positive. So if the solutions of the quadratic equation, which is assuming the left side of Eq. (11) equals 0, are complex, the relation (11) can hold for any $T_{f}$. And if the solutions are real, restriction for $T_{f}$ can be expressed as follows by its solutions $k_{1}, k_{2}$ :

$$
T_{f}<k_{1}, \quad k_{2}<T_{f} \quad\left(k_{1}<k_{2}\right)
$$

The time constant $T_{f}$ is positive, so the time constant $T_{f}$ can be designed to satisfy the following equality:

$$
T_{f}=k_{2}+\varepsilon_{1} \quad\left(\varepsilon_{1}>0\right)
$$

As far as $T_{f}$ satisfies Eq. (13), the looper control system remains stable even if the rolling characteristics change.

\subsection{Sensitivity Reduction Problem}

In order to reduce the influence of the disturbance at low frequency, $F(s)$ is designed such that the sensitivity function $S(s)$ is small at low frequency. The sensitivity function $S(s)$ can be expressed as follows ${ }^{10,11)}$ :

$$
S(s)=\frac{P^{\prime}(s)\left\{1-F(s) P_{M}(s) e^{-L s}\right\}}{P^{\prime}(s)\left\{1-F(s) P_{M}(s) e^{-L^{\prime} s}\right\}+F(s) P_{M}(s) P(s) e^{-L s}}
$$

The sensitivity function value $|S(s)|$ can be expressed as follows:

$$
|S(s)|=\frac{\omega_{n}^{\prime 2} \sqrt{\left(c^{2}+d^{2}\right)\left[\left(\omega_{n}^{2}-\omega^{2}\right)^{2}+\left(2 \zeta \omega_{n} \omega\right)^{2}\right]}}{\sqrt{a^{2}+b^{2}}} \ldots
$$

where

$$
\begin{aligned}
& c=\left(\omega_{M}^{2}-\omega\right)-T_{f} \omega\left(2 \zeta_{M} \omega_{M} \omega\right)-\omega_{M}^{2} \cos L^{\prime} \omega \\
& d=2 \xi_{M} \omega_{M} \omega+T_{f} \omega\left(\omega_{M}^{2}-\omega^{2}\right)+\omega_{M}^{2} \sin L^{\prime} \omega
\end{aligned}
$$


and $a$ and $b$ are parameters related to Eq. (7).

If the time constant $T_{f}$ of the filter $F(s)$ is designed to satisfy Eq. (16), the influence of the disturbance can be reduced below the value of $\alpha(\omega)$.

$$
|S(s)|<\alpha(\omega) \quad \forall \omega
$$

We designed $\alpha$-function as given in Eq. (17) to reduce the plant gain during low frequency. ${ }^{11)}$ The Eq. (17) is the small value $M_{L}$ at low frequency and increases monotonously to the big value $M_{u}$ at high frequency.

$$
\alpha(\omega)=\left|\frac{3.699 M_{L}+2.0256 M_{u} s+M_{u} s^{2}}{3.699+3.0156 s+s^{2}}\right| \ldots \ldots .
$$

where $M_{L}, M_{u}$ are designed by considering the desired performance of the disturbance control. For example, if the influence of the disturbance is designed to reduce $10 \%$ at the frequency of $0.1 \mathrm{rad} / \mathrm{sec}, M_{L}, M_{u}$ are designed as 0.01 and 2.0 respectively.

The Eq. (16) can be rewritten as follows: (see Appendix C)

$$
\begin{aligned}
& \left\{\omega_{n}^{\prime 4}\left[\left(\omega_{n}^{2}-\omega^{2}\right)^{2}+\left(2 \zeta \omega_{n} \omega\right)^{2}\right]\left(p_{2}^{2}+r_{2}^{2}\right)-\alpha^{2}\left(p_{1}^{2}+r_{1}^{2}\right)\right\} T_{f}^{2} \\
& +2\left\{\omega_{n}^{\prime 4}\left[\left(\omega_{n}^{2}-\omega^{2}\right)^{2}+\left(2 \zeta \omega_{n} \omega\right)^{2}\right]\left(p_{2} q_{2}+r_{2} s_{2}\right)\right. \\
& \left.\quad-\alpha^{2}\left(p_{1} q_{1}+r_{1} s_{1}\right)\right\} T_{f} \\
& +\omega_{n}^{\prime 4}\left[\left(\omega_{n}^{2}-\omega^{2}\right)^{2}+\left(2 \zeta \omega_{n} \omega\right)^{2}\right]\left(q_{2}^{2}+s_{2}^{2}\right)-\alpha^{2}\left(q_{1}^{2}+s_{1}^{2}\right)<0
\end{aligned}
$$

$T_{f}$ can be given by $m_{1}$ and $m_{2}\left(m_{1}<m_{2}\right)$, which are the solutions of the quadratic Equation in terms of the time constant $T_{f}$ equating left hand side of Eq. (18) to zero. The second order coefficient in Eq. (18) is positive if $\alpha<1$. And if $\alpha>1$, the second order coefficient is negative. The time constant $T_{f}$ is positive, so we designed $T_{f}$ to satisfy the following inequality (19): (see Appendix C)

$$
0<T_{f}<m_{2} \quad(\text { if } \alpha<1), \quad m_{2}<T_{f} \quad(\text { if } \alpha>1) \text {. }
$$

The time constant $T_{f}$ is designed so that Eqs. (13) and (19) are satisfied. Thus, the looper system can be designed as being adaptive to disturbances satisfying both the robustness and a high accuracy of control performance. The block diagram of the proposed adaptive robust controller is shown in Fig. 4. As shown in the figure, the damping coefficient is estimated in the block "Estimator" using the input/output data of looper system. And using the estimated damping coefficient, $T_{f}$ is calculated by Eqs. (13) and (19). Based on the adapted value of $T_{f}$, the filter $F(s)$ in the robust controller is always maintained most durable for disturbance.

\section{Numerical Experiments}

\subsection{Accuracy of Control}

Numerical simulations are executed to evaluate the performance of the adaptive robust control method.

The condition of numerical simulation is shown in Table 1. The parameter of looper dynamics, $\omega_{n}, \zeta, L, K_{p}, K_{T I}$ are defined by estimating the real plant. ${ }^{5,6)}$ And the parameter

\begin{tabular}{|c|c|c|}
\hline Parameter & value & unit \\
\hline$\omega_{n}$ & 7.0 & $\mathrm{rad} / \mathrm{s}$ \\
\hline$\omega_{n}$, & 7.0 & $\mathrm{rad} / \mathrm{s}$ \\
\hline$\omega_{M}$ & 7. 0 & $\mathrm{rad} / \mathrm{s}$ \\
\hline$\zeta$ & $0.3 \sim 1.5$ & - \\
\hline$\zeta^{\prime}$ & 1. 5 & - \\
\hline$\zeta_{M}$ & 0.7 & - \\
\hline$L$ & $0.2,1.0$ & $\mathrm{~s}$ \\
\hline$L^{\prime}$ & $0.2,1.0$ & $\mathrm{~s}$ \\
\hline$L_{M}$ & 0.2 & $\mathrm{~s}$ \\
\hline $\mathrm{K}_{p}$ & 0.2 & - \\
\hline $\mathrm{K}_{T I}$ & 0.6 & - \\
\hline$T_{f}$ & $0.2 \sim 1.7$ & $\mathbf{s}$ \\
\hline
\end{tabular}
of looper model, $\omega_{n}^{\prime}, \zeta^{\prime}, L^{\prime}$ and the parameter of looper reference model, $\omega_{M}, L_{M}$ are set the same value to $\omega_{n}, \zeta, L$ respectively. The damping coefficient of reference model $\zeta_{M}$

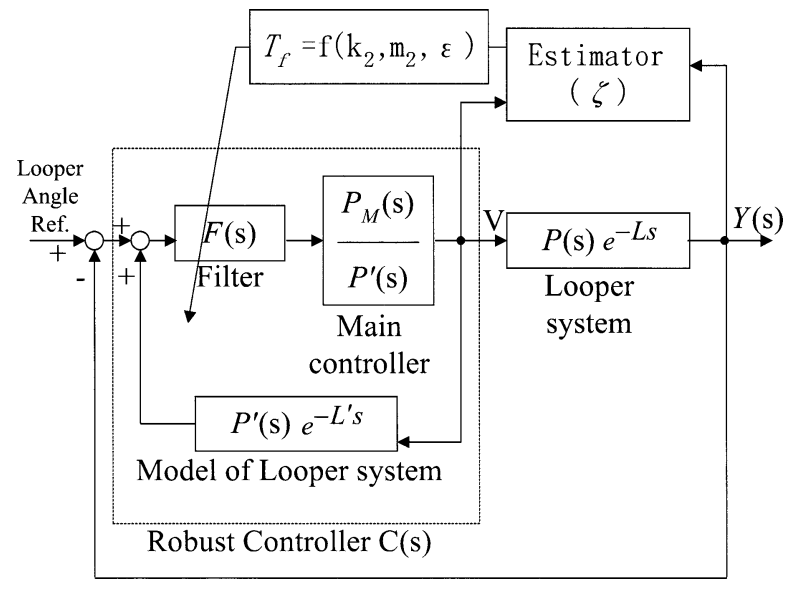

Fig. 4. Adaptive robust control system.

Table 1. Conditions for simulation.

is set 0.7 , which has the most quick response. The gain diagram of $T(s)$ and $S(s)$ are shown in Fig. 5. As shown in Fig. 5 , both the complementary sensitivity function $|T(s)|$ and the sensitivity function $|S(s)|$ are the changing for different damping coefficients. And it confirms that the Eqs. (8) and (16) are satisfied at any frequency. The designed value of the time constant $T_{f}$ is shown in Fig. 6. In the figure, the Eq. (12) is the robust stability condition, and the Eq. (19) is the sensitivity reduction condition. As shown in the figure, the designed value of $T_{f}$ satisfies the conditions in Eqs. (12) and (19).

In the numerical simulations which are the step responses from the stationary state, following two items are considered.

(1) The disturbance corresponding to the change in flow stress of rolled material is given to the looper system. As for the disturbances to the looper system, the sine wave in Eq. (20) and white noise are considered. These disturbances are added on the input of the looper system, $P(s) e^{-L s}$.

$$
D(t)=2.0 \times \sin (0.7 t) \quad(\mathrm{deg})
$$

(2) The damping coefficient is made to change linearly from $\zeta_{1}(=1.5)$ to $\zeta_{2}(=0.3)$ between times $10(\mathrm{~s})$ and $30(\mathrm{~s})$ as shown in Fig. 7.

The simulation results of the internal model control (IMC) with the high constant gain are shown in Fig. 8. Because the gain of the controller is high and constant, the looper height control system becomes unstable after the damping coefficient is changed to small.

The simulation results of the conventional robust control are shown in Fig. 9. In this case, the looper height control 


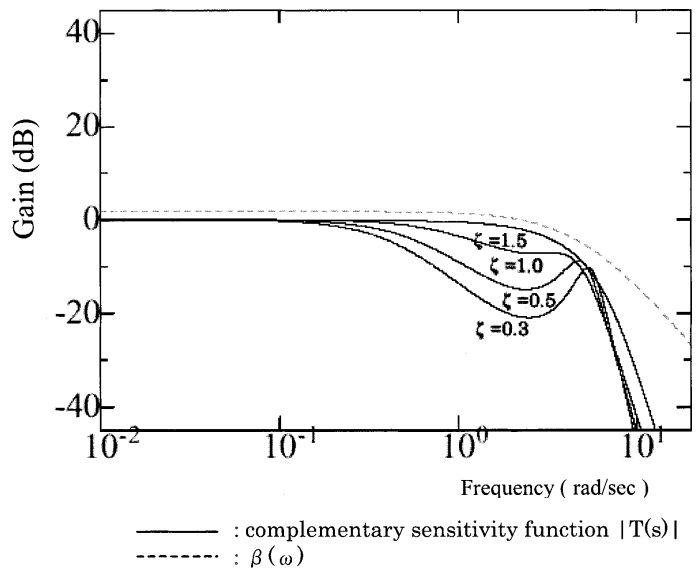

(a) Gain diagram of $\mathrm{T}(\mathrm{s})$ and $\beta$ (s)

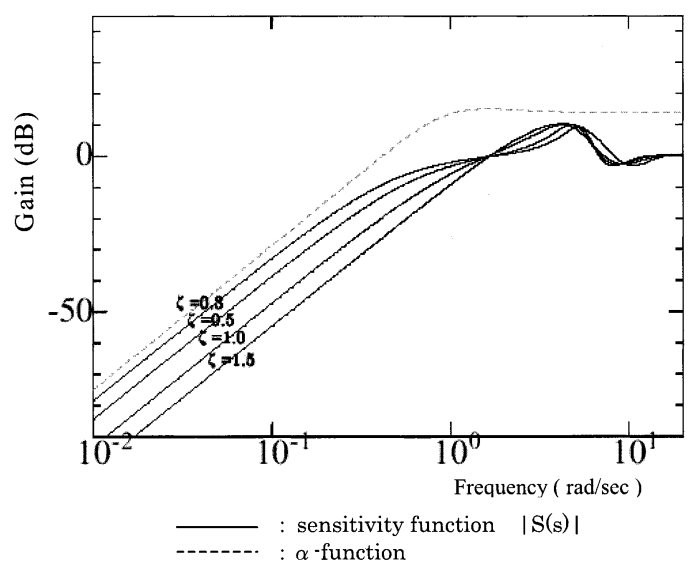

(b) Gain diagram of $\mathrm{S}(\mathrm{s})$ and $\alpha(\mathrm{s})$

Fig. 5. Gain diagram of $T(s)$ and $S(s)$.

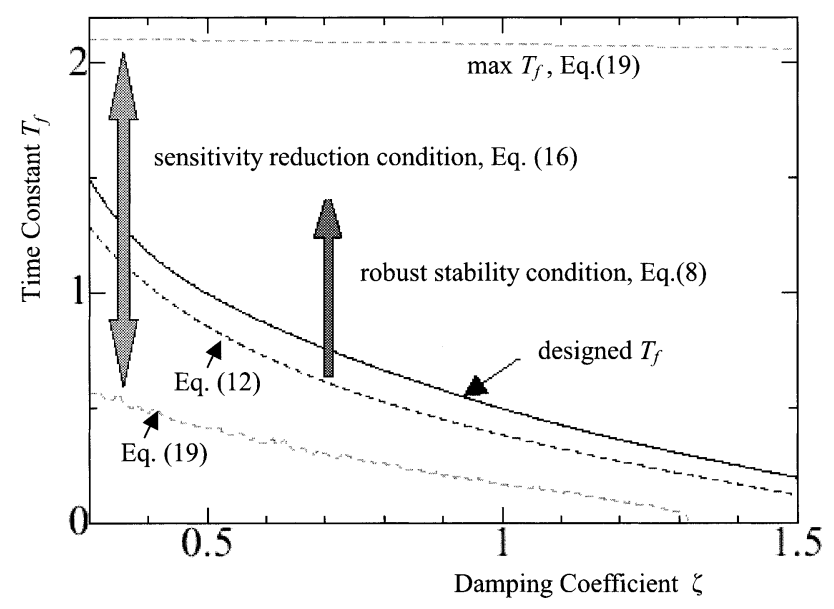

Fig. 6. Condition for $T_{f}$.

system is stabilized regardless of the change in the damping coefficient. However, due to the low constant gain of the controller, the control performance is remained worse than desired. That is, the rise time is longer than that of IMC and the amplitude of looper angle error for the large damping coefficient is larger than that of IMC.

The simulation results of the PI control are shown in Fig. 10. In this case, the looper height control system is stabilized regardless of the change in the damping coefficient, but the control accuracy for the large damping coefficient is

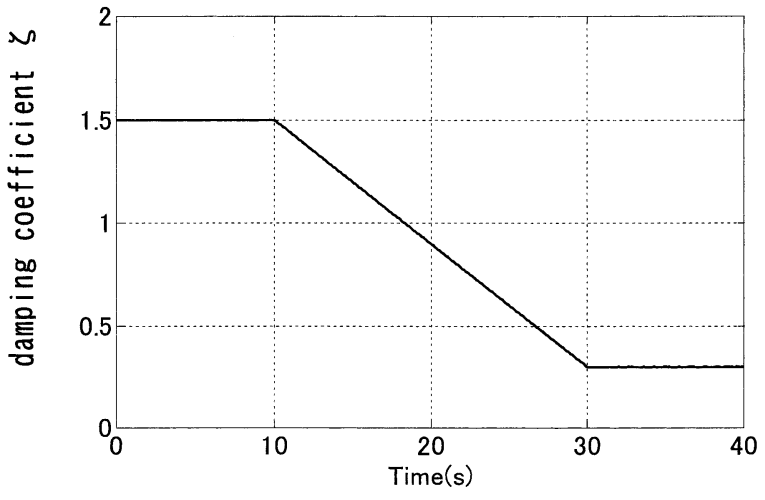

Fig. 7. Damping coefficient in numerical simulation.

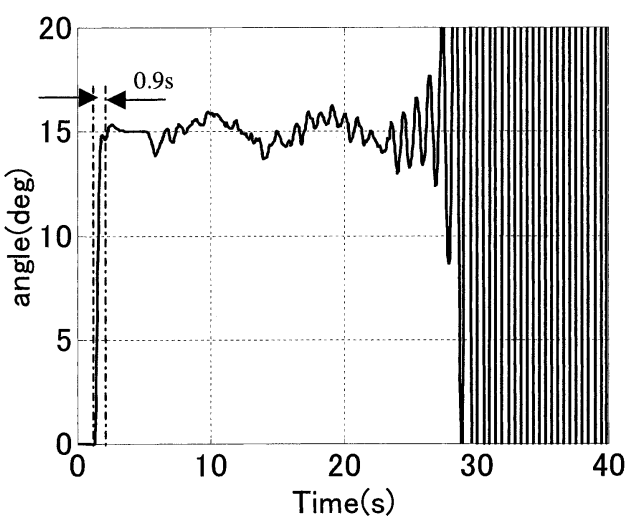

Fig. 8. Simulation result 1 (internal model control with high gain).

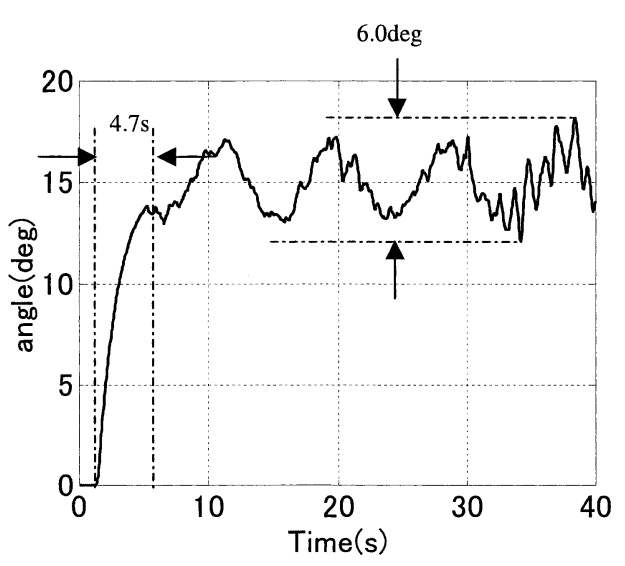

Fig. 9. Simulation result 2 (conventional robust control).

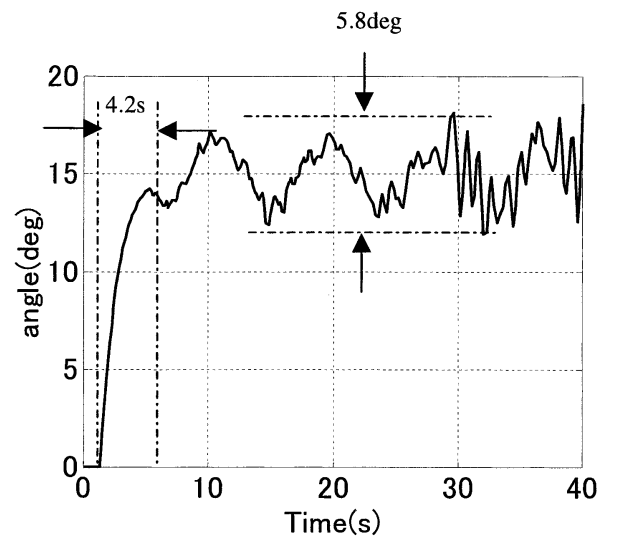

Fig. 10. Simulation result 3 (PI control). 

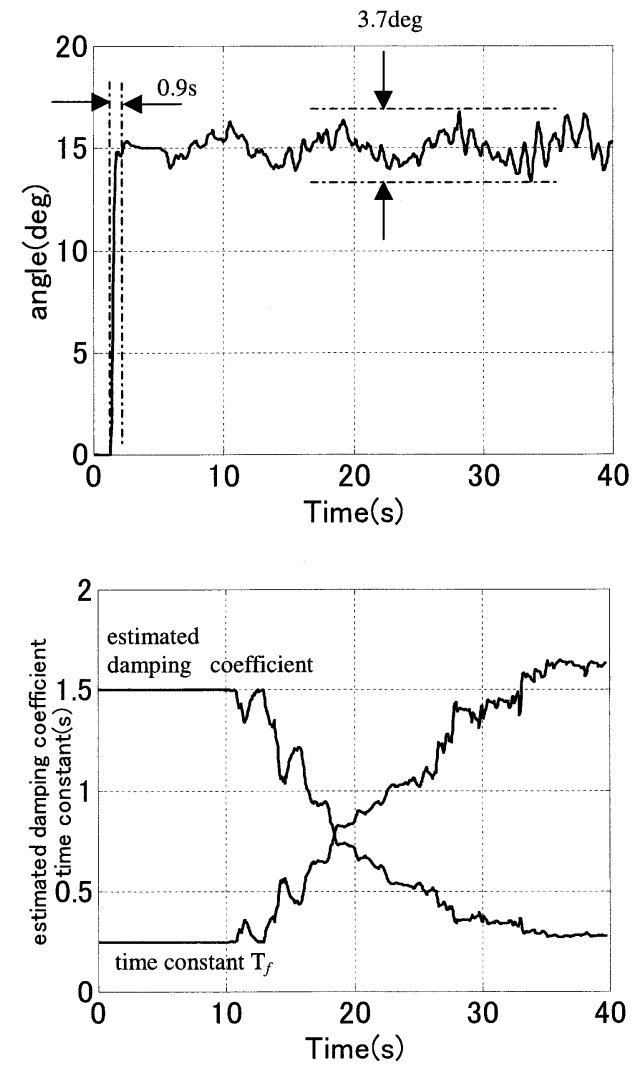

Fig. 11. Simulation result 4 (proposed adaptive robust control).

Table 2. Summary of simulation results.

\begin{tabular}{|c|c|c|}
\hline Control method & rise time & $\begin{array}{c}\text { amplitude } \\
\text { angle error }\end{array}$ \\
\hline $\begin{array}{c}\text { IMC } \\
\text { constant high gain }\end{array}$ & $0.9 \mathrm{sec}$ & $>20 \mathrm{deg}$ \\
\hline $\begin{array}{c}\text { conventional } \\
\text { robust }\end{array}$ & $4.7 \mathrm{sec}$ & $6.0 \mathrm{deg}$ \\
\hline PI control & $4.2 \mathrm{sec}$ & $5.8 \mathrm{deg}$ \\
\hline $\begin{array}{c}\text { adaptive } \\
\text { robust }\end{array}$ & $0.9 \mathrm{sec}$ & $3.7 \mathrm{deg}$ \\
\hline
\end{tabular}

worse than that of IMC.

The simulation results of the proposed adaptive robust control system are shown in Fig. 11. Using the system, while the damping coefficient is not changed to small, the gain of the controller remains at high level. Thus the rise time is shorter than that of the conventional robust controller, and the amplitude of the looper angle error is smaller than these of other controllers. When the damping coefficient is changed to small, the gain of the controller is changed automatically by using the estimation of the damping coefficient. So the response of looper height does not become unstable. That is to say, the proposed adaptive robust controller can guarantee both the stability of the looper height control system and a high degree of accuracy of control.

The comparative summary of simulation results is shown in Table 2. Apparently from this table, the effect of the adaptive robust control system is remarkable in case of these simulations.

\subsection{Effect of the Dead Time}

In the dynamics of the looper control system, there is a dead time due to the CPU calculation time, a mechanical

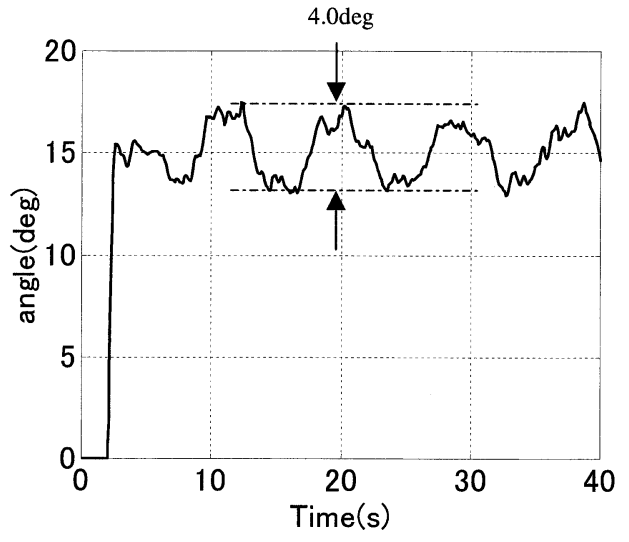

Fig. 12. Simulation result 5 (proposed adaptive robust control: dead time $0.2 \rightarrow 1.0 \mathrm{~s}$ )

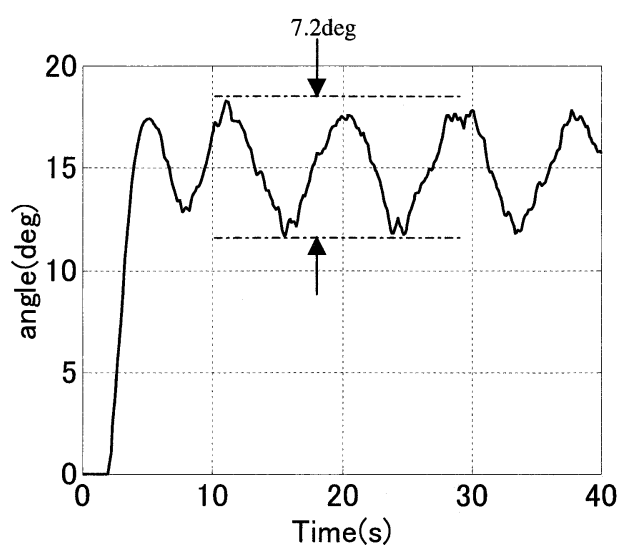

Fig. 13. Simulation result 6 (PI control: dead time $0.2 \rightarrow 1.0 \mathrm{~s}$ ).

play of looper actuators, and so on. The proposed control method is theoretically guaranteed to keep the robust stability for the dead time. In numerical simulations, in order to explain the stability more clearly, the dead time is changed from $0.2(\mathrm{~s})$ to $1.0(\mathrm{~s})$ which are twice or fifth times of the actual dead time. The simulation results of the proposed adaptive robust control system are shown in Fig. 12. In order to compare with them, the simulation results of the PI control are shown in Fig. 13. As shown in the figures, the accuracy of the PI control, in which the dead time is $1.0(\mathrm{~s})$, is worse than the case of Fig. 10 where the dead time is $0.2(\mathrm{~s})$. That is the amplitude of the angle error changes from $5.8(\mathrm{deg})$ to $7.2(\mathrm{deg})$. On the contrary, the results of the proposed robust control have almost the same accuracy in two cases where the dead time are 0.2 (s) in Fig. 11 and 1.0 (s) in Fig. 12. That is the amplitude of the angle error changed to 3.7 (deg) to 4.0 (deg).

That is to say, the proposed adaptive robust controller can guarantee both the system stability and a high degree of accuracy regardless of the dead time in the plant.

\section{Conclusion}

An adaptive robust control method using the estimation of the looper height characteristics has been proposed. Because the conventional robust control determines the gain by considering the worst disturbance, there is a problem of slow response. From lots of actual data, it is confirmed for the looper height system that the damping coeffi- 
cient is greatly changed and contrary to this, both the stationary gain and the resonant frequency are almost constant. In order to solve the problem of slow response of the robust controller, the estimation of the damping coefficient of the looper height characteristic is introduced to adapt the gain of the robust control.

Thus, the adaptive robust control system involving estimator of damping coefficient for looper height dynamics has been developed.

Comparison of the control performance of the proposed adaptive robust controller with that of the conventional control method such as PI control and robust control with fixed gain was made through numerical simulations. It is ascertained that the proposed method has the advantage of both the high degree of accuracy of control and the stability of the looper height control system.

\section{REFERENCES}

1) Y. Kotera and F. Watanabe: Preprints of the 22th Japan Joint Automatic Control Conf., (1979), 305.

2) K. Sekiguchi, Y. Seki, Y. Anbe, K. Fukushima, Y. Tsuji and S. Ueno: Trans. IEE, 108 (1988), No. 1, 31.

3) S. Nakagawa, S. Yagisawa, A. Haraguchi and K. Kimura: Tetsu-toHagané, 78 (1992), T229.

4) Y. Anbe, K. Sekiguchi and H. Imanari: CAMP-ISIJ, 6 (1993), No. 5, 1212.

5) Y. Fujisaki, M. Nakayama, A. Kitamura, M. Konishi and H. Asada: Proc. of the 34th Annual Conf. of the Institute of Systems, Control and Information Engineers, Kyoto, (1990), 321.

6) Y. Fujisaki, A. Kitamura and H. Asada: Trans. Soc. Instrum. Control Eng., 27 (1991), No .6, 723.

7) S. Nishino, H. Narasaki, A. Kitamura, Y. Fujita and H. Asada: Trans. Inst. Systems, Control Information Eng., 9, (1996), No. 11, 548.

8) S. Sagara, K. Akizuki, T. Nakamizo and T. Katayama: SisutemuDoutei, Soc. Instrum. Control Eng., (1983).

9) M. Morari and E. Zafiriou: Robust Process Control, Prentice Hall, New Jersey, (1989), 39.

10) A. Fujimori: Robust Control, Corona Publishing Co., Ltd., Tokyo, (2001), 119.

11) M. Saeki: Trans. Inst. Systems, Control Information Eng., 42 (1998), No. 1, 26.

\section{Appendix A}

The complementary sensitive function $T(s)$ and the sensitive function $S(s)$ in Fig. 3 can be expressed as follows ${ }^{8,9)}$ :

$$
\begin{aligned}
& T(s)=\frac{C(s) P(s) e^{-L s}}{1+C(s) P(s) e^{-L s}} \\
& S(s)=\frac{1}{1+C(s) P(s) e^{-L s}}
\end{aligned}
$$

$C(s)$ is given in Eq. (3), so $T(s)$ and $S(s)$ can be derived out as follows:

$$
\begin{aligned}
T(s) & =\frac{\frac{F(s) P_{M}(s) P(s) e^{-L s}}{P^{\prime}(s)\left\{1-F(s) P_{M}(s) e^{-L^{\prime} s}\right\}}}{1+\frac{F(s) P_{M}(s) P(s) e^{-L s}}{P^{\prime}(s)\left\{1-F(s) P_{M}(s) e^{-L^{\prime} s}\right\}}} \\
= & \frac{F(s) P_{M}(s) P(s) e^{-L s}}{P^{\prime}(s)\left\{1-F(s) P_{M}(s) e^{-L^{\prime} s}\right\}+F(s) P_{M}(s) P(s) e^{-L s}}
\end{aligned}
$$

$$
\begin{aligned}
S(s) & =\frac{1}{1+\frac{F(s) P_{M}(s) P(s) e^{-L s}}{P^{\prime}(s)\left\{1-F(s) P_{M}(s) e^{-L^{\prime} s}\right\}}} \\
& =\frac{P^{\prime}(s)\left\{1-F(s) P_{M}(s) e^{-L^{\prime} s}\right\}}{P^{\prime}(s)\left\{1-F(s) P_{M}(s) e^{-L^{\prime} s}\right\}+F(s) P_{M}(s) P(s) e^{-L s}}
\end{aligned}
$$

\section{Appendix B}

The Eq. (B-1) can be expressed by the Eqs. (7), (8):

$$
\frac{\omega_{M}^{2} \omega_{n}^{2} \sqrt{\left(\omega_{n}^{\prime 2}-\omega^{2}\right)^{2}+\left(2 \zeta^{\prime} \omega_{n}^{\prime} \omega\right)^{2}}}{\sqrt{a^{2}+b^{2}}}<\beta .
$$

where $\beta=|\beta(\omega)|$.

$$
\begin{aligned}
& \therefore \quad \beta^{2}\left(a^{2}+b^{2}\right)>\left(\omega_{M}^{2} \omega_{n}^{2}\right)^{2}\left[\left(\omega_{n}^{\prime 2}-\omega^{2}\right)^{2}+\left(2 \zeta^{\prime} \omega_{n}^{\prime} \omega\right)^{2}\right] . \\
& \therefore \quad \beta^{2}\left[\left(p_{1} T_{f}+q_{1}\right)^{2}+\left(r_{1} T_{f}+s_{1}\right)^{2}\right] \\
& \quad>\left(\omega_{M}^{2} \omega_{n}^{2}\right)^{2}\left[\left(\omega_{n}^{\prime 2}-\omega^{2}\right)^{2}+\left(2 \zeta^{\prime} \omega_{n}^{\prime} \omega\right)^{2}\right] \ldots \ldots \ldots . . .
\end{aligned}
$$

where $p_{1}=-\omega_{n}^{2} \omega\left[2 \zeta_{M} \omega_{M} \omega\left(\omega_{n}^{2}-\omega^{2}\right)+2 \zeta \omega_{n} \omega\left(\omega_{M}^{2}-\omega^{2}\right)\right]$

$$
\begin{aligned}
q_{1}= & \omega_{n}^{2}\left[\left(\omega_{M}^{2}-\omega^{2}\right)\left(\omega_{n}^{2}-\omega^{2}\right)-4 \zeta_{M} \omega_{M} \zeta \omega_{n} \omega^{2}\right] \\
& -\omega_{n}^{\prime 2} \omega_{M}^{2}\left[\left(\omega_{n}^{2}-\omega^{2}\right) \cos L^{\prime} \omega+2 \zeta \omega_{n} \omega \sin L^{\prime} \omega\right] \\
& +\omega_{n}^{2} \omega_{M}^{2}\left[\left(\omega_{n}^{\prime 2}-\omega^{2}\right) \cos L \omega+2 \zeta^{\prime} \omega_{n}^{\prime} \omega \sin L \omega\right] \\
r_{1}= & \omega_{n}^{\prime 2} \omega\left[\left(\omega_{M}^{2}-\omega^{2}\right)\left(\omega_{n}^{2}-\omega^{2}\right)-4 \zeta_{M} \omega_{M} \zeta \omega_{n} \omega^{2}\right] \\
s_{1}= & 2 \omega_{n}^{\prime 2}\left[\zeta_{M} \omega_{M} \omega\left(\omega_{n}^{2}-\omega^{2}\right)+\zeta \omega_{n} \omega\left(\omega_{M}^{2}-\omega^{2}\right)\right] \\
& -\omega_{n}^{\prime 2} \omega_{M}^{2}\left[-\left(\omega_{n}^{2}-\omega^{2}\right) \sin L^{\prime} \omega+2 \zeta \omega_{n} \omega \cos L^{\prime} \omega\right] \\
& +\omega_{n}^{2} \omega_{M}^{2}\left[-\left(\omega_{n}^{\prime 2}-\omega^{2}\right) \sin L \omega+2 \zeta^{\prime} \omega_{n}^{\prime} \omega \cos L \omega\right]
\end{aligned}
$$

The Eq. (B-3) can be expressed as follows by rewriting in terms of $T_{f}$ :

$$
\begin{aligned}
\therefore \quad \beta^{2} & \left(p_{1}^{2}+r_{1}^{2}\right) T_{f}^{2}+2 \beta^{2}\left(p_{1} q_{1}+r_{1} s_{1}\right) T_{f} \\
& +\beta^{2}\left(q_{1}^{2}+s_{1}^{2}\right)-\left(\omega_{M}^{2} \omega_{n}^{2}\right)^{2}\left\{\left(\omega_{n}^{\prime 2}-\omega^{2}\right)+\left(2 \zeta^{\prime} \omega_{n}^{\prime 2} \omega\right)^{2}\right\}>0
\end{aligned}
$$

The solution of the Eq. (B-5) are $k_{1}$ and $k_{2}$, which can be expressed as Eqs. (B-6), (B-7).

$$
\begin{aligned}
& \beta^{2}\left(p_{1}^{2}+r_{1}^{2}\right) T_{f}^{2}+2 \beta^{2}\left(p_{1} q_{1}+r_{1} s_{1}\right) T_{f} \\
& \left.\quad+\beta^{2}\left(q_{1}^{2}+s_{1}^{2}\right)-\left(\omega_{M}^{2} \omega_{n}^{2}\right)^{2}\left\{\omega_{n}^{\prime 2}-\omega^{2}\right)^{2}+\left(2 \zeta^{\prime} \omega_{n}^{\prime 2} \omega\right)^{2}\right\}=0
\end{aligned}
$$

$$
\begin{aligned}
& k_{1}=\left\{-\beta^{2}\left(p_{1} q_{1}+r_{1} s_{1}\right)-\left[\beta^{4}\left(p_{1} q_{1}+r_{1} s_{1}\right)^{2}\right.\right. \\
& -\beta^{2}\left(p_{1}^{2}+r_{1}^{2}\right)\left\{\beta^{2}\left(q_{1}^{2}+s_{1}^{2}\right)-\left(\omega_{M}^{2} \omega_{n}^{2}\right)^{2}\left[\left(\omega_{n}^{\prime 2}-\omega^{2}\right)^{2}\right.\right. \\
& \left.\left.\left.\left.+\left(2 \zeta^{\prime} \omega_{n}^{\prime} \omega\right)^{2}\right]\right\}\right]^{1 / 2}\right\} / \beta^{2}\left(p_{1}^{2}+r_{1}^{2}\right) \\
& k_{2}=\left\{-\beta^{2}\left(p_{1} q_{1}+r_{1} s_{1}\right)+\left[\beta^{4}\left(p_{1} q_{1}+r_{1} s_{1}\right)^{2}\right.\right. \\
& -\beta^{2}\left(p_{1}^{2}+r_{1}^{2}\right)\left\{\beta^{2}\left(q_{1}^{2}+s_{1}^{2}\right)-\left(\omega_{M}^{2} \omega_{n}^{2}\right)^{2}\left[\left(\omega_{n}^{\prime 2}-\omega^{2}\right)^{2}\right.\right. \\
& \left.\left.\left.\left.+\left(2 \zeta^{\prime} \omega_{n}^{\prime} \omega\right)^{2}\right]\right\}\right]^{1 / 2}\right\} / \beta^{2}\left(p_{1}^{2}+r_{1}^{2}\right)
\end{aligned}
$$

\section{Appendix C}

The Eq. (C-1) can be expressed by the Eqs. (15), (16): 


$$
\begin{gathered}
\frac{\omega_{n}^{\prime 2} \sqrt{\left(c^{2}+d^{2}\right)\left[\left(\omega_{n}^{2}-\omega^{2}\right)^{2}+\left(2 \zeta \omega_{n} \omega\right)^{2}\right.}}{\sqrt{a^{2}+b^{2}}}<\alpha \ldots . .(\mathrm{C}-1) \\
\therefore \quad \omega_{n}^{\prime 4}\left\{\left(c^{2}+d^{2}\right)\left[\left(\omega_{n}^{2}-\omega^{2}\right)^{2}+\left(2 \zeta \omega_{n} \omega\right)^{2}\right]\right\}<\alpha^{2}\left(a^{2}+b^{2}\right) \\
\therefore \quad \omega_{n}^{\prime 4}\left[\left(\omega_{n}^{2}-\omega^{2}\right)^{2}+\left(2 \zeta \omega_{n} \omega\right)^{2}\right]\left[\left(p_{2} T_{f}+q_{2}\right)^{2}+\left(r_{2} T_{f}+s_{2}\right)^{2}\right] \\
\quad<\alpha^{2}\left[\left(p_{1} T_{f}+q_{1}\right)^{2}+\left(r_{1} T_{f}+s_{1}\right)^{2}\right] \\
\text { where } \quad p_{2}=-\omega\left(2 \zeta_{M} \omega_{M} \omega\right) \\
q_{2}=\left(\omega_{M}^{2}-\omega^{2}\right)-\omega_{M}^{2} \cos L^{\prime} \omega \\
r_{2}=\omega\left(\omega_{M}^{2}-\omega^{2}\right) \\
s_{2}=2 \zeta_{M} \omega_{M} \omega+\omega_{M}^{2} \sin L^{\prime} \omega
\end{gathered}
$$

and $p_{1}, q_{1}, r_{1}, s_{1}$ are parameters related to Eq. (B-3).

The Eq. (C-3) can be expressed by putting in order $T_{f}$ as follows:

$$
\begin{aligned}
& \left\{\omega_{n}^{\prime 4}\left[\left(\omega_{n}^{2}-\omega^{2}\right)^{2}+\left(2 \zeta \omega_{n} \omega\right)^{2}\right]\left(p_{2}^{2}+r_{2}^{2}\right)-\alpha^{2}\left(p_{1}^{2}+r_{1}^{2}\right) T_{f}^{2}\right. \\
& +2\left\{\omega_{n}^{\prime 4}\left[\left(\omega_{n}^{2}-\omega^{2}\right)^{2}+\left(2 \zeta \omega_{n} \omega\right)^{2}\right]\left(p_{2} q_{2}+r_{2} s_{2}\right)\right. \\
& \left.\quad-\alpha^{2}\left(p_{1} q_{1}+r_{1} s_{1}\right)\right\} T_{f} \\
& +\omega_{n}^{\prime 4}\left[\left(\omega_{n}^{2}-\omega^{2}\right)^{2}+\left(2 \zeta \omega_{n} \omega\right)^{2}\right]\left(q_{2}^{2}+s_{2}^{2}\right)-\alpha^{2}\left(q_{1}^{2}+s_{1}^{2}\right)<0
\end{aligned}
$$

The solution of the Eq. (C-5) are $m_{1}$ and $m_{2}$, which can be expressed as Eqs. (C-6), (C-7).

$$
\begin{aligned}
& \left\{\omega_{n}^{\prime 4}\left[\left(\omega_{n}^{2}-\omega^{2}\right)^{2}+\left(2 \zeta \omega_{n} \omega\right)^{2}\right]\left(p_{2}^{2}+r_{2}^{2}\right)-\alpha^{2}\left(p_{1}^{2}+r_{1}^{2}\right)\right\} T_{f}^{2} \\
& +2\left\{\omega_{n}^{\prime 4}\left[\left(\omega_{n}^{2}-\omega^{2}\right)^{2}+\left(2 \zeta \omega_{n} \omega\right)^{2}\right]\left(p_{2} q_{2}+r_{2} s_{2}\right)\right. \\
& \left.\quad-\alpha^{2}\left(p_{1} q_{1}+r_{1} s_{1}\right)\right\} T_{f} \\
& +\omega_{n}^{\prime 4}\left[\left(\omega_{n}^{2}-\omega^{2}\right)^{2}+\left(2 \zeta \omega_{n} \omega\right)^{2}\right]\left(q_{2}^{2}+s_{2}^{2}\right)-\alpha^{2}\left(q_{1}^{2}+s_{1}^{2}\right)=0
\end{aligned}
$$

$$
\begin{aligned}
& m_{1}=\frac{-B-\sqrt{B^{2}-C A}}{A} \\
& m_{2}=\frac{-B+\sqrt{B^{2}-C A}}{A}
\end{aligned}
$$

where

$$
\begin{aligned}
& A=\omega_{n}^{\prime 4}\left[\left(\omega_{n}^{2}-\omega^{2}\right)^{2}+\left(2 \zeta \omega_{n} \omega\right)^{2}\right]\left(p_{2}^{2}+r_{2}^{2}\right)-\alpha^{2}\left(p_{1}^{2}+r_{1}^{2}\right) \\
& B=\omega_{n}^{\prime 4}\left[\left(\omega_{n}^{2}-\omega^{2}\right)^{2}+\left(2 \zeta \omega_{n} \omega\right)^{2}\right]\left(p_{2} q_{2}+r_{2} s_{2}\right)-\alpha^{2}\left(p_{1} q_{1}+r_{1} s_{1}\right) \\
& C=\omega_{n}^{\prime 4}\left[\left(\omega_{n}^{2}-\omega^{2}\right)^{2}+\left(2 \zeta \omega_{n} \omega\right)^{2}\right]\left(q_{2}^{2}+s_{2}^{2}\right)-\alpha^{2}\left(q_{1}^{2}+s_{1}^{2}\right)
\end{aligned}
$$

The following relation is always satisfied analytically.

$$
\omega_{n}^{\prime 4}\left[\left(\omega_{n}^{2}-\omega^{2}\right)^{2}+\left(2 \zeta \omega_{n} \omega\right)^{2}\right]\left(p_{2}^{2}+r_{2}^{2}\right)=p_{1}^{2}+r_{1}^{2}
$$

The sign of second order coefficient in Eq. (C-4) is changed by $\alpha$. That is , if $\alpha>1$, the sign of second order coefficient is negative. If $\alpha<1$, the sign of one is positive. So the Eq. (C-4) can be expressed as follows by the solutions $m_{1}, m_{2}$ :

$$
\begin{aligned}
& m_{1}<T_{f}<m_{2} \quad(\alpha<1) \\
& T_{f}<m_{1}, \quad m_{2}<T_{f} \quad(\alpha>1)
\end{aligned}
$$

